\title{
Cosmic star formation history: high redshift galaxies and black holes
}

\author{
Jacqueline Bergeron \\ Institut d'Astrophysique de Paris-CNRS-UPMC, 98bis Boulevard Arago, \\ F-75014, Paris, France \\ email: bergeron@iap.fr
}

\begin{abstract}
We review the current status of searches for high redshift galaxies and quasars, together with their derived luminosity function, star formation history and relative contribution to the UV ionizing background. These searches have been carried out in the optical and nearinfrared with the HST for very deep pencil beam surveys and ground-based telescopes for deep large area surveys. The peak of galaxy and black hole formation activity occurs at $z \sim 2-3$, with a strong decrease in UV luminosity density in the redshift interval $z \sim 3$ to 6 . This decrease seems to extend beyond $z \sim 6$ for luminous galaxies, as there are only a few $z \sim 7-8$ secure galaxy candidates. The star formation rate at $z \sim 6$ is dominated by $0.1 \mathrm{~L}^{\star}$ galaxies. The $z \gtrsim 5$ samples for both types of sources are too small to constrain the faint end of their luminosity function or even its knee. Consequently, predictions of the number density of $z \gtrsim 7$ galaxies is highly uncertain; their search with JWST and ELTs should cover areas of at least $\sim 1 \mathrm{deg}^{2}$ and will require long campaigns ( $\gtrsim 1$ month). Faint quasars of small black hole mass should also be detected in these surveys. Searches for $z \sim 10$ rare, bright quasars and higher $z$ transient sources, gamma-ray bursts and population III SNe, should be undertaken with dedicated facilities. The physical properties of high $z$ galaxies and the metal-enrichment of the intergalactic medium at early epochs will be determined by high-resolution spectroscopic observations with ELTs requiring substantial exposure times $(\sim 100 \mathrm{hr})$.
\end{abstract}

Keywords. Cosmology: observations; Galaxies: evolution; Quasars: general.

\section{Introduction}

Important steps in our knowledge of high redshift galaxies and quasars were made possible by deep surveys with space- and ground-based facilities. Luminosity functions (LFs) and star formation history of Lyman break galaxies (LBGs) have been derived at $2 \lesssim z \lesssim 5$, as well as the cosmic evolution of the LF bright end of quasars. However, a number of questions are still open due to the limited depth of the current surveys with 8-10 m class telescopes.

The break of the LBG LF is not or ill-defined at $z \gtrsim 4$. Thus, it is not possible to ascertain whether or not the shape of the LBG LF follows a Schechter function. The cosmic evolution of $L^{\star}(\mathrm{LBG})$ is not known either. For high $z$ quasars, a rare population of objects, the depth of the current surveys, especially the Sloan digital sky survey (SDSS), prevents reaching the break of the quasar's LF. At $z \gtrsim 5$, the fraction of quasars among the LBG populations cannot be derived, as done at $2 \lesssim z \lesssim 4$, due to the small sizes of the samples.

Although many $z \sim 6$ LBGs, Ly- $\alpha$ emitting galaxies and bright quasars have been discovered, there is not yet any spectroscopically confirmed object at $z \gtrsim 7$. Such objects are crucial to constrain the ionization level of the intergalactic medium at the end of the reionization epoch and determine which population (faint quasars, bright starbursts or low luminosity LBGs) provides the main radiative source for the reionization of the 
universe. It is expected that the UKIRT infrared deep sky survey (UKIDSS) will soon detect $z \sim 7$ quasars. The recent discovery of a $z=6.3$ gamma-ray burst (GRB) by the Swift satellite is very promising since the GRBs should outshine the quasars at $z \sim 8-10$.

The existence of massive quasars and galaxies at very high $z$ is uncertain mainly due to the short time available for the growth of black holes and galaxy mass assembly: $\Delta t=(4.5$ and 2.9$) \times 10^{8}$ yr for $6<z<10$ and $10<z<20$, respectively (adopting $\Omega_{\Lambda}, \Omega_{\mathrm{m}}, h=0.7$, 0.3, 70 throughout this paper). Explosive events, GRBs and population III supernovae, may thus be best candidates for probing the reionization epoch. Their identification implies specific search strategies with dedicated facilities and a fast infrared spectroscopic follow-up with large telescopes.

We will now investigate in more details the problems outlined above and the questions that should be solved with extremely large ground-based telescopes (ELTs).

\section{Galaxies : number and size}

The deep surveys conducted with the HST (pioneered by R. Williams) have demonstrated that the confusion limit is far from being reached even at the highest depths achieved so far. This is a consequence of the substantial decrease of galaxy sizes with increasing $z$. Bouwens et al. (2004a) have determined the cosmic evolution of the galaxy mean half-light radius, which roughly follows a power-law $\mathrm{r}_{\mathrm{hl}} \propto(1+z)^{-1.05}$ (see Fig. 1).

At $z \sim 6$, a radius $\mathrm{r}_{\mathrm{hl}}=1.0 \mathrm{kpc}$ corresponds to an angular size of 170 milli-arcsecond (mas). Extrapolating the above power-law to higher $z$ leads to $\mathrm{r}_{\mathrm{hl}}(z=10)=0.6 \mathrm{kpc}=$ 150 mas, i.e. sizes far larger than the spatial resolution of ELTs at their diffraction limit ( 2 and 7 mas at $\lambda=1 \mu \mathrm{m}$ for 100 and $30 \mathrm{~m}$ telescopes, respectively).

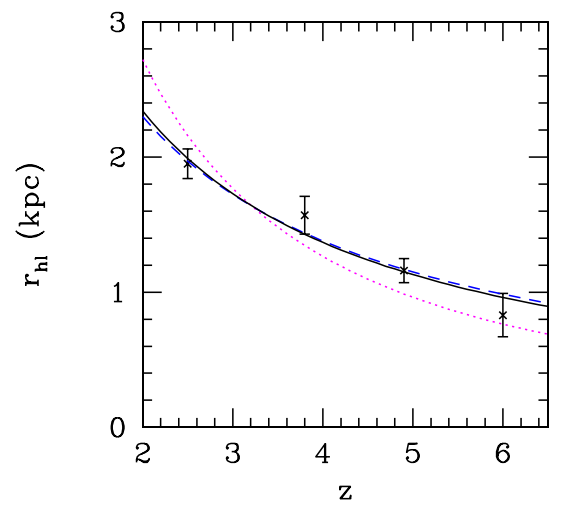

Figure 1. Mean half-light radius versus redshift for objects of a fixed luminosity (from Bouwens et al. 2004a, astro-ph/0406562).

The number density of bright $z \sim 6$ LBG candidates in "large" area surveys $\left(\simeq 0.2 \mathrm{deg}^{2}\right)$ with the Subaru telescope is $1.6 \times 10^{-2} \operatorname{arcmin}^{-2}$ down to $\mathrm{z}_{\mathrm{B}, \mathrm{AB}}=25.4$ (Shimasaku et al. 2005). The ultra-deep surveys of $z \sim 6$ galaxies with the HST yield a number density of 1.4 i-dropouts $\operatorname{arcmin}^{-2}$ down to $\mathrm{i}_{775, \mathrm{AB}}=28.5$ (or $15 \mathrm{nJy}$ ) with significant cosmic variance between sub-fields (Bouwens et al. 2004a; Dickinson et al. 2004). Spectroscopic identification of such faint objects at a resolution of 1000-3000 and signal-to-noise S/N $>10$ could only be achieved with ELTs (see Table 1). 


\section{Galaxy luminosity function}

\subsection{Galaxies at $z \sim 3-6$}

The $z \sim 3$ LBG luminosity function (LF) is well fit by a Schechter function with parameters $\Phi^{\star}=4.4 \times 10^{-3} h^{3} \mathrm{Mpc}^{-3} \mathrm{mag}^{-1}, \mathrm{~m}^{\star}=24.5\left(\right.$ or $\left.\mathrm{M}^{\star}=-21.1\right)$ and $\alpha=-1.6$ (Adelberger \& Steidel 2000). The $z \sim 4$ LBG LF roughly follows the one at $z \sim 3$ (see Fig. 2), whereas the $z \sim 5$ samples are too small to constrain the bright end of their LF or even $L^{\star}$ (Ouchi et al. 2004). Nevertheless, there is a clear decrease of the luminosity density from $z \sim 3-4$ to $z \sim 5$-6 (Bunker et al. 2004; Bouwens et al. 2004a; Stanway et al. 2005): assuming identical LF parameters as at $z \sim 3, \Phi^{\star}$ decreases by nearly an order of magnitude from $z \sim 3$ to $z \sim 6$. Although a luminosity evolution cannot be ruled out, Bouwens et al. (2004a) favor a density evolution scaling as $(1+z)^{-2.8}$.

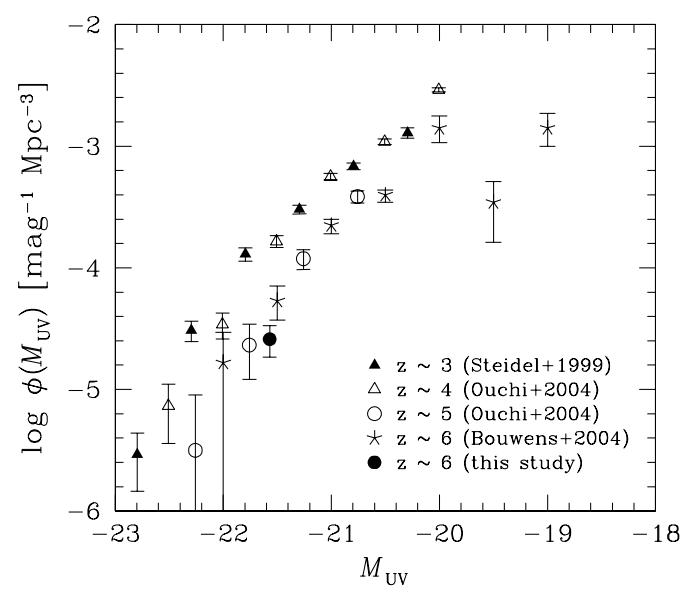

Figure 2. FUV luminosity functions of LBGs (from Shimasaku et al. 2005, astro-ph/0504373).

The observed FUV luminosity density of bright, $\mathrm{M}_{\mathrm{FUV}}<-21.3$, LBGs evolves drastically with redshift with a peak at $z \sim 3$ (Shimasaku et al. 2005). It decreases by $\sim 10$ from $z \sim 3$ to $z \sim 6$ (see Fig. 3). This bright magnitude limit is close to $\mathrm{M}^{\star}$ of $z \sim 3$ LBGs. At high $z$ the evolution of fainter LBGs $\left(\mathrm{M}_{\mathrm{FUV}}<-19\right.$ or $\left.0.15 L_{z=3}^{\star}\right)$ is much milder. The LBG star formation rate can be derived from their FUV luminosity density. At $z \sim 6$, the star formation rate density of fainter LBGs exceeds that of brighter LBGs by a factor of $\sim 30$.

The validity of a Schechter LF fit for LBGs could be questioned as the bright end is not well constrained. The SDSS enables the discovery of bright starburst galaxies at $z \sim 2.5$. A small sample of such galaxies, 4-5 magnitudes brighter than $\mathrm{M}^{\star}$ LBGs, yields a large excess over the extrapolated Schechter LF of $z \sim 3$ LBGs, as shown in Fig. 4 (Bentz et al. 2004). A K-correction to move this sample to $z \sim 3$ gives a power-law fit for the LF bright end, as obtained for quasars, with a slope $\beta(\mathrm{L}) \simeq-2.9$. However, only low resolution SDSS spectra are available and the nature of these starburst galaxies is uncertain. Indeed quasars are also selected by LBG colour selection techniques, as already outlined by Steidel et al. (2002). Higher resolution spectroscopic follow-up studies have shown that all the SDSS selected bright starbursts belong to a class of rare, weak BAL 


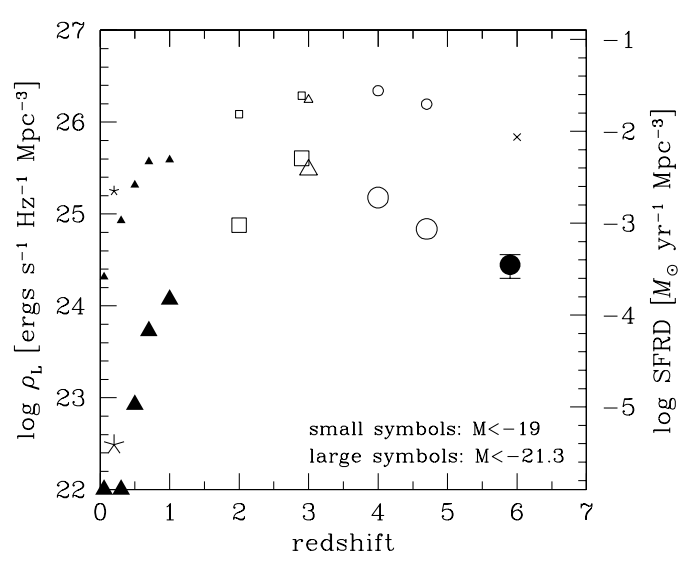

Figure 3. Cosmic evolution of the LBG FUV luminosity density (from Shimasaku et al. 2005, astro-ph/0504373).

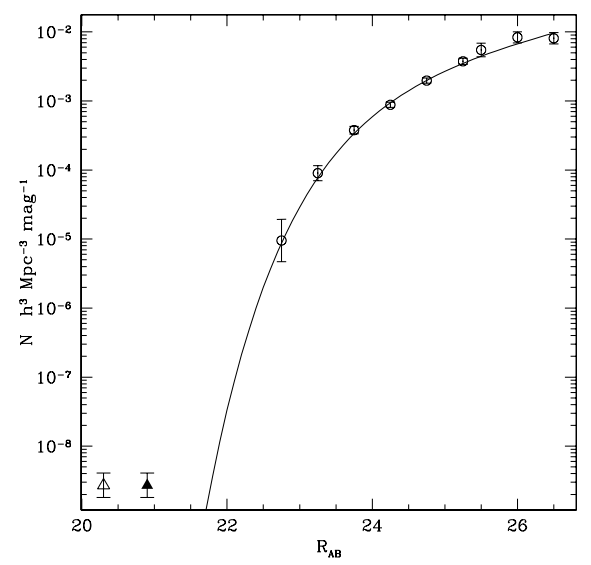

Figure 4. Luminosity function of starburst galaxies at $z \sim 3$ (from Bentz et al. 2004, astro-ph/0309641).

quasars (Appenzeller et al. 2005; Ivison et al. 2005). Constraining the shape of high $z$ LBGs is thus still an open issue.

\subsection{Galaxies at $z \sim 7-10$}

Searches for $z \gtrsim 7$ galaxies have mainly been made with the HST in the Hubble ultra deep field (UDF) and the GOODS fields observed with the ACS and NICMOS. The Z 850 UDF dropouts are detected in a $5.8 \operatorname{arcmin}^{2}$ NICMOS mosaic down to $H_{1.6 \mu \mathrm{m}, \mathrm{AB}} \sim 27.5$, or $\sim 0.3 L_{z=3}^{\star}$. There are five detections of which four secure $z \sim 7.5$ candidates (Bouwens et al. 2004b), thus a number density $n \sim 0.7 \operatorname{arcmin}^{-2}$. These galaxies are highly clustered: of the five sources mentioned above, four fall roughly within a one $\operatorname{arcmin}^{2}$ field. Assuming no evolution at high redshift $(z \geqslant 3.8)$ would lead to an expected number of $14, z \sim 7.5$ candidates. The estimated luminosity density (integrated down to $0.3 L_{z=3}^{\star}$ ) 


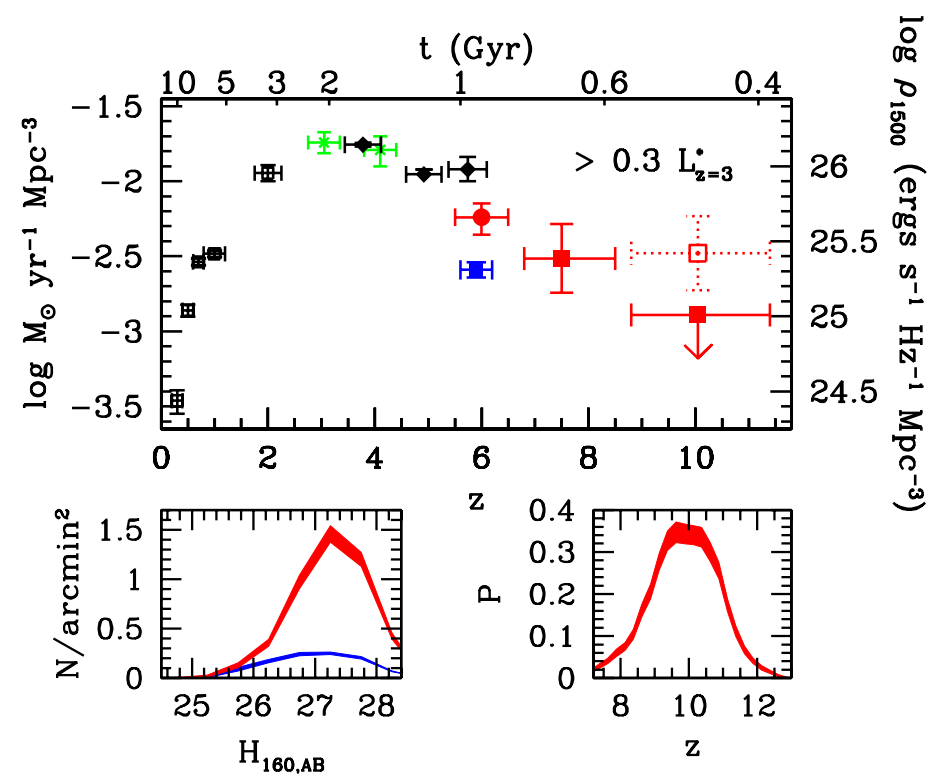

Figure 5. Cosmic star formation rate versus redshift (from Bouwens et al. 2005, astro-ph/0503116).

is $1 / 5$ that found at $z \sim 3.8$ (see Fig. 5): this is consistent with a significant evolution at the bright end of the LF from $z \sim 3.8$ to $z \sim 7.5$ and, possibly, also an evolution of $L^{\star}$.

Using all NICMOS bands, Bouwens et al. (2005) have searched for $J_{1.1 \mu \mathrm{m}, \mathrm{AB}}$ dropouts down to $H_{1.6 \mu \mathrm{m}, \mathrm{AB}} \lesssim 28$. Among the detected sources, there are at most three possible $z \sim 10$ candidates but those could be low-redshift interlopers. In the latter case, there would be further evolution from $z \sim 6-7$ to $z \sim 10$, as shown in Fig. 5 .

Searches have also been carried out for high $z$ lensed galaxies by several teams $(\mathrm{Hu}$ et al. 2002; Kneib et al. 2004; Egami et al. 2005). There are a few secure candidates detected with NICMOS at $z \sim 7-8$ down to $J_{1.1 \mu \mathrm{m}, \mathrm{AB}} \sim 24.5$. One $z \sim 10$ candidate with VLT-ISAAC imaging and spectroscopy (Pelló et al. 2004) has been infirmed by further studies (Weatherley et al. 2004; Lehnert et al. 2005; Smith et al. 2006).

\section{Predicted surface density of high $z$ galaxies}

The nature and properties of the sources responsible for the reionization of the universe at $z>6$ are not yet well constrained by the observations. Stiavelli et al. 2004 have explored models characterized by essentially two parameters in addition to an assumed Schechter luminosity function: the Lyman continuum escape fraction, $f_{c}$ and the clumpiness of the intergalactic medium, $C$. The derived surface density of ionizing sources is not strongly sensitive to the duration of the reionization epoch, $\Delta z$, and nearly independent of the terminal redshift of reionization, $z_{f}$ (for $z_{f}<10$ ).

Their results are presented in Fig. 6 for $z_{f}=6, \Delta z=1$ and different LF parameters: a unique value of the faint end slope, $\alpha=-1.6$, and various $M_{1400}^{\star}:-23.2,-21.2$ (which is roughly the $\left.\mathrm{M}_{1400}^{\star} a t z=3\right),-17.5$, and -13.5 , i.e. from bright starbursts to low mass, faint dwarf galaxies. The predicted number of sources at $\mathrm{m}_{\mathrm{AB}}=29$ for $z_{f}>6$ and $\Delta z=1$ is 


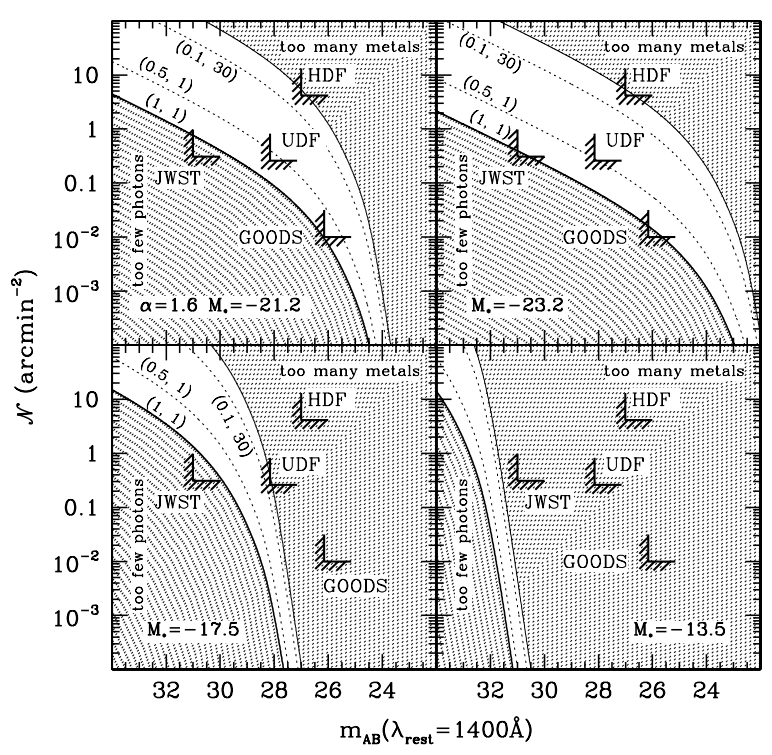

Figure 6. Surface density of reionization sources versus apparent AB magnitude for luminosity functions with different knees (from Stiavelli et al. 2004, astro-ph/0309835).

$n=1$ and $0.2 \operatorname{arcmin}^{-2}$ for $\mathrm{M}^{\star}=-21.2$ and -17.3 , respectively. These faint sources could be detected with JWST and ELTs (see Table 1) and these surveys should cover several tens of $\operatorname{arcmin}^{2}$. If the ionizing sources are much fainter, $-17.5<\mathrm{M}^{\star}<-13.5$, larger area surveys (several $\mathrm{deg}^{2}$ ) will be needed to uncover the rare sources with $\mathrm{m}_{\mathrm{AB}} \sim 29-30$.

\section{High $z$ quasars}

The peak of the quasar-AGN activity occurs at $z \sim 2-3$. At higher $z(\gtrsim 5)$, bright quasars are rare and the fainter quasar-AGN population is not well constrained. Thus, the relative contribution of galaxies and quasars to the UV luminosity density at early epochs is still an open issue.

The search for $z \sim 6$ quasars by the SDSS team down to $\mathrm{z}_{\mathrm{AB}}=20.2\left(\right.$ or $\mathrm{M}_{1400}=-26.4$ ) yields a very small number of sources, $n \simeq 2 \times 10^{-3} \mathrm{deg}^{-2}$ (Fan et al. 2001, 2003). The number of faint quasars at intermediate redshift is derived from LBG surveys, since the colour selection used uncover both the LBG and quasar populations. At $2 \lesssim z \lesssim 4$, the fraction of quasars among the LBG samples (down to $R_{\mathrm{AB}}=25.5$ at $z \sim 3$ ) is $3 \%$ (Steidel et al. 1999, 2003, 2004). At $z \sim 3$, the same result is obtained when combining X-ray data with LBG colour selection (Nandra et al. 2005).

\subsection{Evolution of the quasar spatial density}

The SDSS $z \sim 6$ quasars have emission line and continuum properties similar to those at lower $z$, thus high gas metallicity and large black hole masses (Fan et al. 2001). From $z=0.5-2$ (2dF survey) to $z \simeq 6$, the comoving density of luminous quasars $\left(\mathrm{M}_{1400}<\right.$ -26.7 ) decreases as $n \propto 10^{-0.5 z}$, thus by $\sim 30$ as shown in Fig. 7 (Fan et al. 2004). This confirms earlier results for SDSS quasar samples at $3.6<z<5.0$ with $\mathrm{M}_{\mathrm{B} \text {,rest }}<-26.0$, 


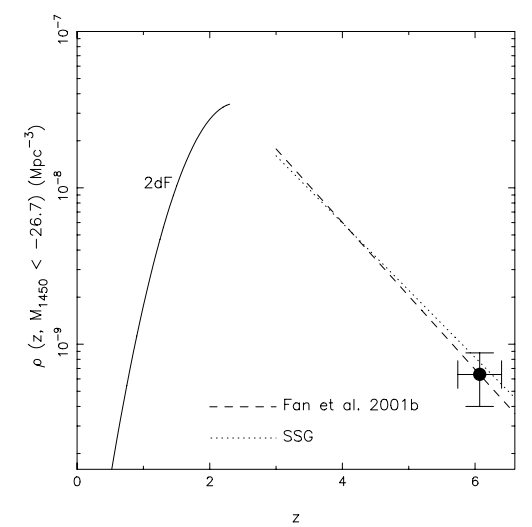

Figure 7. Evolution of the quasar comoving spatial density at $\mathrm{M}_{1450}<-26.7$ in the $\Lambda$ model (from Fan et al. 2004, astro-ph/0405138).

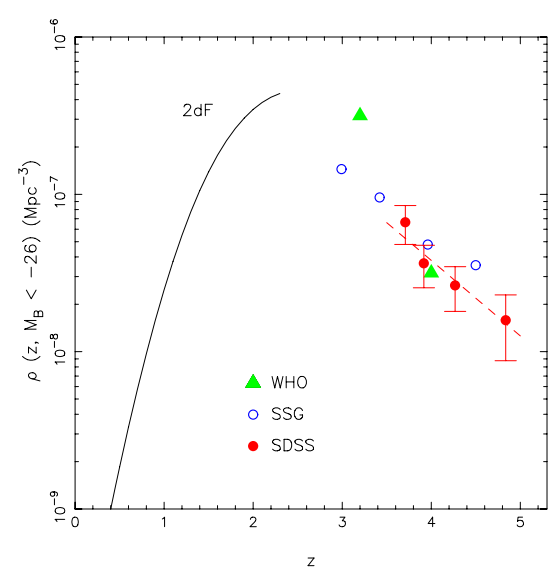

Figure 8. Cosmic evolution of the quasar luminosity spatial density at $\mathrm{M}_{\mathrm{B} \text { (rest) }}<-26.0$ (from Fan et al. 2001, astro-ph/0008123).

or $\mathrm{M}_{1450}<-25.5$, shown in Fig. 8 together with multicolour (WHO) and grism (SSG) quasar samples (Fan et al. 2001).

Over the range $3 \lesssim z \lesssim 6$, the decrease in spatial density of LBGs with $\mathrm{M}_{1450}<$ $-21.6<\mathrm{M}^{\star}$ is much milder, only a factor of $\sim 6$ (Shimasaku et al. 2005; Bouwens et al. 2004a). That for faint quasars in the same $z$ range is unknown due to the small sizes of the $z \sim 5$-6 LBG samples.

\subsection{Quasar luminosity density and luminosity function}

The large COMBO-17 survey yields a large sample of quasars at $1.2<z<4.8$ to a $R=24$ limit (Wolf et al. 2003). The quasar peak activity is indeed at $z \sim 2-3$ as can be seen in Fig. 9. At $z \simeq 2.7$, the UV luminosity density of quasars with $\mathrm{M}_{1450}<-24$ is 0.5 times the one of LBGs with $\mathrm{M}_{1450}<-21.5$. The Lyman continuum escape fraction of quasars equals $0.5-1.0$ as compared to about 0.2 for the bluest quartile of LBGs (Steidel et al. 2001). The value for the bulk of the LBGs is not well known and could be smaller (Shapley et al. 2003). Consequently, at $z \sim 3$ the quasars should be the main contributors to the UV ionizing background. 


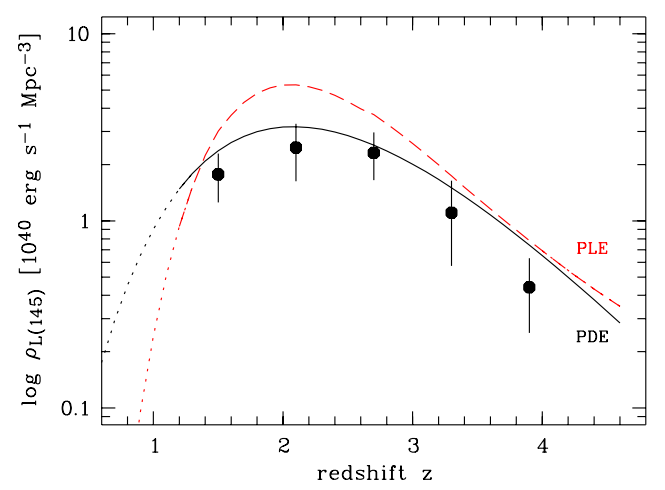

Figure 9. Cosmic evolution of faint AGN UV luminosity density (from Wolf et al. 2003, astro-ph/0304072).

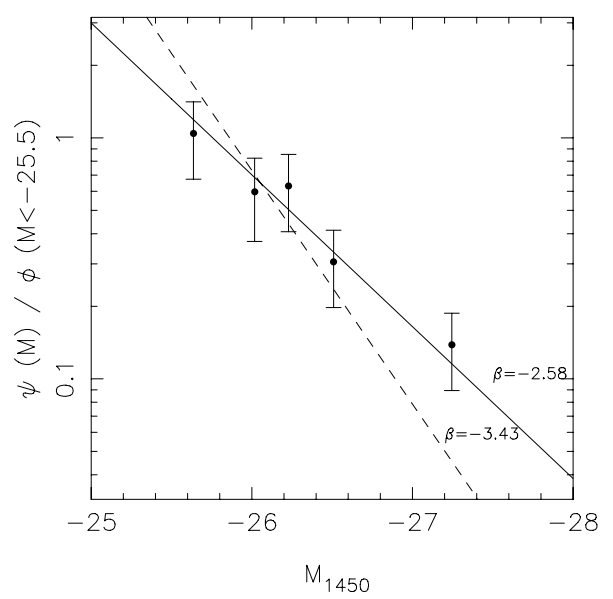

Figure 10. Bright end of the quasar luminosity function (from Fan et al. 2001, astro-ph/0008123).

Despite the size of the COMBO-17 sample, the shape of the quasar's LF is uncertain: there is a mild curvature but no sharp break. This sample cannot help distinguishing between a pure density (PDE) and pure luminosity (PLE) evolution of the quasar LF.

The SDSS quasar samples enables a determination of their LF's bright end. A powerlaw fit gives a slope $\beta \simeq-3.4$ at $z<2.1$ and $\beta \simeq-2.6$ at $z \sim 4$ (Fan et al. 2001; see Fig. 10). This flattening is not fully confirmed by the SDSS $z \sim 6$ quasar sample, although the uncertainty on the slope at these higher $z$ is large, $\Delta \beta=0.7$ (Fan et al. 2004).

The search for fainter $z \sim 6$ quasars may help constraining the evolution of the LF's bright end. A quasar survey was undertaken with the multi-colour CFHT Legacy Survey (CFHTLS). The data of the four deep fields $\left(4 \times 1 \mathrm{deg}^{2}\right)$ were used to build a sample of i-dropouts to a limit $\mathrm{z}_{\mathrm{AB}}=23.3$, or $\mathrm{M}_{1400}=-23.5$ (Willott et al. 2005). Near-infrared photometry reveals that all the 24 quasar candidates at $5.7<z<6.4$ are low-mass stars, including $\mathrm{L}$ and $\mathrm{T}$ dwarfs. This negative result can be used to either constrain the LF's bright end slope or check whether the fraction of quasars among LBG samples 
could still be of $\sim 3 \%$ at high $z$. The non-detection of $z \sim 6$ faint quasars, $n\left(\mathrm{z}_{\mathrm{AB}}>\right.$ $23.3)<0.25 \mathrm{deg}^{-2}$, can be compared to the observed surface density of SDSS quasars, $n\left(\mathrm{z}_{\mathrm{AB}}>20.2\right) \simeq 2 \times 10^{-3} \mathrm{deg}^{-2}$. Following Schirber \& Bullock (2003), we assume that the break in the quasar LF occurs at $\mathrm{M}_{1400}>-23.5$. This gives a constraint on the LF's bright end slope, $\beta(z \sim 6)<-1.7$, which would imply a further flattening of the LF with increasing $z$. Alternatively, the break could occur at $-26.8<\mathrm{M}_{1400}<-23.5$. Comparison to $z \sim 6$ bright LBG samples gives the magnitude of faint quasars for an expected surface density $n=0.25 \mathrm{deg}^{-2}$ and a $3 \%$ fraction of quasars among LBG samples. We assume a Schechter LBG LF with $\mathrm{M}_{1450}^{\star}=-21.1$ and a faint end slope $\alpha=-1.6$, as at $z=3$. Given the number surface density of Subaru LBGs, $n\left(\mathrm{z}_{884, \mathrm{AB}}<25.4\right)=57 \mathrm{deg}^{-2}$ (Shimasaku et al. 2005), the expected magnitude of quasars with a surface density of 0.25 per deg $^{2}$ is $\mathrm{z}_{\mathrm{AB}}=23.9$, i.e. fainter than Willott et al.'s magnitude limit. For $\mathrm{M}_{1450}^{\star}(z=6)>$ $\mathrm{M}_{1450}^{\star}(z=3)$ and the same surface density, the expected quasar $\mathrm{z}_{\mathrm{AB}}$ magnitudes are larger than 24. The current $z \sim 6$ faint quasar surveys are thus consistent with an unevolving fraction of quasars among LBG samples.

\section{Quasars, GRBs and population III SNe at the reionization epoch}

\subsection{Quasars at $z \sim 7-15$}

Massive black holes $(\mathrm{BHs})$ at $z \sim 6$ are a rare population of objects. Are massive BHs already in place at $z \sim 10$ ? Assuming that the SDSS $z \sim 6$ quasars are emitting at the Eddington limit, their $\mathrm{BH}$ masses are $\sim(1-5) \times 10^{9} \mathrm{M}_{\odot}$. The mass of their progenitors can be estimated assuming an accretion efficiency of 0.15 and Eddington accretion rate; the derived masses are $\mathrm{M}_{\mathrm{BH}}=(1-5) \times 10^{6} \mathrm{M}_{\odot}$ at $z \sim 10$ and a few $10^{3} \mathrm{M}_{\odot}$ at $z \sim 20-30$. Open issues are the possible efficient merging of thousands of high $z, 10^{3} \mathrm{M}_{\odot}$ BHs or the existence of primordial BHs.

The expected number density of $10^{6}-10^{9} \mathrm{M}_{\odot}$ BHs at $z \sim 8-10$ is anyway very small, which raises the problem of their search strategy. Results of on-going near-infrared surveys, as e.g. UKIDSS, will help defining future high $z$ quasar searches with JWST and ELTs. The expected magnitude of $z \sim 10 \mathrm{BHs}$ with masses of a few $10^{5} \mathrm{M}_{\odot}$ are $J_{\mathrm{AB}} \sim 29$ $K_{\mathrm{AB}} \sim 28$.

Luminous sources are best suited to study the metal-enrichment of the intergalactic medium during reionization and the clustering of the active star-formation sites from their absorption signatures (e.g. O I, C II, C IV) in the flux of background objects. Detection limits for spectroscopy at a resolution $\mathrm{R}=2000$ are given in $\S 7$. For the same parameters as in Table $1(\mathrm{R}=2000, \mathrm{~S} / \mathrm{N}=20, \Delta \mathrm{t}=50 \mathrm{hr})$, and a point source observed with a $100 \mathrm{~m}$ ELT, the minimun $\mathrm{BH}$ masses corresponding to the detection limit $J_{\mathrm{AB}}=29.1$ $(8.3 \mathrm{nJy})$ and $H_{\mathrm{AB}}=28.2(19 \mathrm{nJy})$ are $\mathrm{M}_{\mathrm{BH}}=(1.5$ and 5$) \times 10^{5} \mathrm{M}_{\odot}$ at $z \sim 9$ and 12 , respectively. Thus, we can expect that there will be quasars bright enough, at least up to $z \sim 9-10$, for intermediate resolution spectroscopic studies to probe the early evolution of the intergalactic medium.

\subsection{GRBs and population III SNe}

Although also rare, GRBs are bright objects, currently identified up to $z=6.3$ (see e.g. Haislip et al. 2005; Tagliaferri et al. 2005). They are even better suited than quasars (that they should outshine at $z \sim 8$-10) to study the "metal intergalactic forest". From the known GRB samples, we estimate that the afterglow fluxes of an average GRB should decrease from $K_{\mathrm{AB}}=23.6(1.5 \mu \mathrm{Jy})$ to $K_{\mathrm{AB}}=27(0.05 \mu \mathrm{Jy})$ at 1 to 10 days after 
explosion. The most luminous GRBs are 20 times brighter, thus their fluxes are equal to $1.5 \mu \mathrm{Jy} 10$ days after explosion. This sets the time lag for ELT spectroscopic follow-up.

Spectroscopic obervations at high resolution, $\mathrm{R}=1 \times 10^{4}$, can easily be undertaken with ELTs. For $1.5 \mu \mathrm{Jy}$ sources, a $\mathrm{S} / \mathrm{N}=40$ will be reached for exposure times $\Delta \mathrm{t}=1.8$ and $15 \mathrm{hr}$ for 100 and $30 \mathrm{~m}$ telescopes, respectively.

Population III SNe should exist at the onset of reionization, when the metallicity was less than $10^{-4}$ solar (e.g. Heger et al. 2001; Bromm et al. 2003). Stars with masses in the range 140-260 $\mathrm{M}_{\odot}$ will explode as a pair-instability supernova. Population III stars with masses outside this narrow interval should collapse into BHs. The peak of the light curve $(1.5$ to $5 \mu \mathrm{m})$ of population III SNe should last for about one month. Their predicted magnitudes in the near-infrared are $\mathrm{m}_{\mathrm{AB}} \sim 25-26$ at $z \sim 20$. For a source with $K_{\mathrm{AB}}=25.2$ $(0.3 \mu \mathrm{Jy})$ at $z \sim 16$, spectroscopic observations at a resolution $\mathrm{R}=1 \times 10^{4}$ and a $\mathrm{S} / \mathrm{N}=$ 40 can be achieved with a $100 \mathrm{~m}$ ELT in $\Delta \mathrm{t}=14 \mathrm{hr}$. Studies at lower resolution, $\mathrm{R}=$ 2000, can be carried out with 30 m ELTs.

\section{Detection limits with future facilities}

We use the exposure time calculators available for JWST and ESO-ELT, to estimate magnitude limits for imaging and spectroscopic modes. Results are presented in Table 1 for given $\mathrm{S} / \mathrm{N}$ and exposure time $\Delta t$.

Table 1. Detection limits

\begin{tabular}{cccc}
\hline telescope & JWST & $30 \mathrm{~m}$ & $100 \mathrm{~m}$ \\
\hline \hline imaging & point source & diffraction limit & $\mathrm{S} / \mathrm{N} \sim 7, \Delta \mathrm{t}=1 \mathrm{hr}$ \\
\hline magnitude & $J_{\mathrm{AB}} \sim K_{\mathrm{AB}} \sim 29$ & $J_{\mathrm{AB}} \sim 29$ & $J_{\mathrm{AB}} \sim 31.5, K_{\mathrm{AB}} \sim 30$ \\
FoV & $2.2^{\prime} \times 4.4^{\prime}$ & $\Phi \sim 2^{\prime}(\mathrm{AO} / 2 \mu \mathrm{m})$ & $\Phi \sim 2^{\prime}(\mathrm{AO} / 2 \mu \mathrm{m})$ \\
Res (mas) & 35 & 7 & 2.1 \\
\hline Spectroscopy & extended source & $\mathrm{R} \sim 2000,25$ mas & $\mathrm{S} / \mathrm{N} \sim 20, \Delta \mathrm{t}=50 \mathrm{hr}$ \\
& $\mathrm{R} \sim 100,100$ mas & $\mathrm{R} \sim 2000,25 \mathrm{mas}$ \\
\hline magnitude & $\mathrm{AB}(2.6 \mu \mathrm{m}) \sim 27$ & $J_{\mathrm{AB}}-1 \sim K_{\mathrm{AB}} \sim 26.7$ & $J_{\mathrm{AB}}-1 \sim K_{\mathrm{AB}} \sim 28.0$ \\
\hline Spectroscopy & point source & diffraction limit & $\mathrm{S} / \mathrm{N} \sim 50, \Delta \mathrm{t}=100 \mathrm{hr}$ \\
R $\sim 10^{4}$ & & & \\
\hline magnitude & & $R_{\mathrm{AB}} \sim 25.5$ & $R_{\mathrm{AB}} \sim 28.5, \mathrm{~K}_{\mathrm{AB}} \sim 26.0$ \\
\hline
\end{tabular}

\section{Future searches of high $z$ sources}

Deep surveys undertaken in the near-infrared to a $\mathrm{m}_{\mathrm{AB}} \sim 29$ limit and covering at least one $\operatorname{deg}^{2}$ should detect a fairly large number of $z \sim 7-10$ galaxies. The spatial density of these sources strongly depends on a possible evolution of their LF (see $\S 4$ ). If the non-evolution observed at $3 \lesssim z<5$ extends beyond $z \sim 6$, i.e. $\mathrm{M}_{1400}^{\star}(z \sim 10)=-21.2$ or $J_{\mathrm{AB}} \sim 27.3$, one expects a few $10^{3}$ high $z$ galaxies per $\operatorname{deg}^{2}$. However, this number density could easily be an order of magnitude smaller if there is a significant evolution of the LF, in particular a strong decrease of $\mathrm{L}^{\star}$. As clustering may be present, as is the case at $z \sim 6-7$, several fields, each a fraction of $\mathrm{deg}^{2}$, should be observed.

A one month campaign with JWST will be needed to cover one $\operatorname{deg}^{2}$ to a $\mathrm{m}_{\mathrm{AB}} \sim 29$ limit. The pixel scale of JWST (35 mas) is well matched to the expected size of $z \sim 10$ 
galaxies (150 mas). Similar surveys could be carried out with ELTs; they will require a similar ( $\gtrsim 40 \mathrm{~m}$ ELT) or somewhat larger (30m ELT) total observing time. Moderate adaptive correction would be necessary, together with a pixel scale larger than the high spatial resolution at the diffraction limit.

The spectroscopic follow-up of high $z$ galaxies at a resolution of a few $10^{3}$ should be undertaken with ELTs to determine their physical properties, e.g. kinematics, metallicity. Multi-integral-field-unit spectrographs with a field of view of several $\operatorname{arcmin}^{2}$, and possibly $\mathrm{OH}$ suppressor, are foreseen. Long exposure times will be needed for $J_{\mathrm{AB}} \sim 27-28$ sources as shown in Table 1.

Discovery of bright high $z$ quasars, GRBs and population III SNe will require dedicated ground- and space- base telescopes (rare sources \& transient objects). Spectroscopy of lower mass $\mathrm{BHs}, \mathrm{M} \sim 10^{6} \mathrm{M}_{\odot}$ at $z \sim 10$, could be performed at a resolution of a few $10^{3}$. GRBs and population III SNe and, if they exist, quasars with high mass BHs can be observed at a much higher resolution, $\mathrm{R} \gtrsim 10^{4}$, to probe the metal enrichment of the intergalactic medium and the clustering of metal-rich sites, up to the onset of the reionization of the universe.

\section{References}

Adelberger, K.L. \& Steidel, C.C. 2000, ApJ 544, 218

Appenzeller, I., Stahl, O., Tapken, C., Mehlert, D. \& Noll, S. 2005, A\&A 435, 465

Bentz, M.C., Osmer, P.S. \& Weinberg, D.H. 2004, ApJ 600, L19

Bouwens, R.J., Illingworth, G.D., Blakeslee, J.P., Broadhurst, T.J. \& Franx, M. 2004a, ApJ 611, L1

Bouwens, R.J., Thompson, R.I., Illingworth, G.D., Franx, M., van Dokkum, P.G., Fan, X., Dickinson, M.E., Eisenstein, D.J. \& Rieke, M.J. 2004b, ApJ 616, L79

Bouwens, R.J., Illingworth, G.D., Thompson, R.I. \& Franx, M. 2005, ApJ 624, L5

Bromm, V., Yoshida, N. \& Hernquist, L. 2003, ApJ 596, L138

Bunker, A.J., Stanway, E.R., Ellis, R.S. \& McMahon, R.G. 2004, MNRAS 355, 374

Dickinson, M., Stern, D., Giavalisco, M., Ferguson, H.C., Tsvetanov, Z. et al. 2004, ApJ 600, L99

Egami, E., Kneib, J.-P., Rieke, G.H., Ellis, R.S., Richard, J. et al. 2005, ApJ 618, L5

Fan, X., Strauss, M.A., Schneider, D.P., Gunn, J.E., Lupton, R.H. et al. 2001, AJ 121, 54

Fan, X., Strauss, M.A., Schneider, D.P., Becker, R.H., White, R.L. et al. 2003, AJ 125, 1649

Fan, X., Hennawi, J.F., Richards, G.T., Strauss, M.A., Schneider, D.P. et al. 2004, AJ 128, 515

Haislip, J., Nysewander, M., Reichart, D., Levan, A., Tanvir, N. et al. 2005, Nature in press, astro-ph/0509660

Heger, A., Woosley, S. E., Baraffe, I. \& Abel, T. 2001, astro-ph/0112059

Hu, E.M., Cowie, L.L., McMahon, R.G., Capak, P., Iwamuro, F., Kneib, J.-P., Maihara, T. \& Motohara, K. 2002, ApJ 568, L75

Ivison, R.J., Smail, I., Bentz, M., Stevens, J.A., Menndez-Delmestre, K., Chapman, S.C. \& Blain, A.W. 2005, MNRAS 362, 535

Kneib, J.-P., Ellis, R.S., Santos, M.R. \& Richard, J. 2004, ApJ 607, 697

Lehnert, M.D., Förster Schreiber, N.M. \& Bremer, M.N. 2005, ApJ 624, 80

Nandra, K., Laird, E.S. \& Steidel, C.C. 2005, MNRAS 360, L39

Ouchi, M., Shimasaku, K., Okamura, S., Furusawa, H., Kashikawa, N. et al. 2004, ApJ 611, 660

Pelló, R., Schaerer, D., Richard, J., Le Borgne, J.-F. \& Kneib, J.-P. 2004, A\&A 416, L35

Schirber, M. \& Bullock, J.S. 2003, ApJ 610, 105

Shapley, A.E., Steidel, C.C., Pettini, M. \& Adelberger, K.L. 2003, ApJ 588, 65

Shimasaku, K., Ouchi, M., Furusawa, H., Yoshida, M., Kashikawa, N. \& Okamura, S. 2005, PASJ 57, 447

Smith, G.P., Sand, D.J., Egami, E., Stern, D. \& Eisenhardt, P.R. 2006, ApJ 636, 575

Stanway, E.R., McMahon, R.G. \& Bunker, A.J. 2005, MNRAS 359, 1184 
Steidel, C.C., Adelberger, K.L., Giavalisco, M., Dickinson, M. \& Pettini, M. 1999, ApJ 519, 1

Steidel, C.C., Pettini, M. \& Adelberger, K.L. 2001, ApJ 546, 665

Steidel, C.C., Hunt, M.P., Shapley, A.E., Adelberger, K.L., Pettini, M., Dickinson, M. \& Giavalisco, M. 2002, ApJ 576, 653

Steidel, C.C., Adelberger, K.L., Shapley, A.E., Pettini, M., Dickinson, M. \& Giavalisco, M. 2003, ApJ 592, 728

Steidel, C.C., Shapley, A.E., Pettini, M., Adelberger, K.L., Erb, D.K., Reddy, N.A. \& Hunt, M.P. 2004, ApJ 604, 534

Stiavelli, M., Fall, S.M. \& Panagia, N. 2004, ApJ 600, 508

Tagliaferri, G., Antonelli, L.A., Chincarini, G., Fernandez-Soto, A., Malesani, D. et al. 2005, AESA Letters in press, astro-ph/0509766

Weatherley, S.J., Warren, S.J. \& Babbedge, T.S.R. 2004, A\&A 428, L29

Willott, C.J., Delfosse, X., Forveille, T., Delorme, P. \& Gwyn, S.D.J. 2005, ApJ 633, 630

Wolf, C., Wisotzki, L., Borch, A., Dye, S., Kleinheinrich, M. \& Meisenheimer, K. 2003, AE $A$ 408,499 